CASE REPORT

\title{
First Case of Azithromycin Resistance in Salmonella Typhi, Isolated in a Patient with Prosthetic Valve Replacement
}

\author{
Arif Maqsood Ali ${ }^{1}$, Agha Babar Hussain ${ }^{2}$, Azhar Mehmood Kiyani ${ }^{3}$ \\ ${ }^{1}$ Department of Pathology and Blood Bank, Rawalpindi Institute of Cardiology, Rawalpindi, Pakistan \\ ${ }^{2}$ Department of Pathology and Blood Bank, Rawalpindi Institute of Cardiology, Rawalpindi, Pakistan \\ ${ }^{3}$ Department of Medicine/Cardiology, Rawalpindi Institute of Cardiology, Rawalpindi, Pakistan
}

\begin{abstract}
Salmonella enterica serovar Typhi (S. Typhi) is one of the typhoidal Salmonellae that causes typhoid fever. We report the first case of an XDR $S$. Typhi in a suspected case of Endocarditis with Aortic Valve Replacement and deranged INR. The identification was carried out by an automated system. The antibiogram was performed according to CLSI recommendations. The XDR $S$. Typhi isolates were found resistant to Azithromycin. Although XDR S. Typhi has been reported in our country and elsewhere, however, till to date no complete resistant isolate of $S$. Typhi to Azithromycin has been reported in Pakistan. The patient had a smooth recovery on intravenous meropenem. The case highlights the importance of the acquisition of resistance to the last line antibiotics in S. Typhi in our country and is of concern to infectious disease specialists to ensure infection control to avoid its spread. J Microbiol Infect Dis 2021; 11(1):32-35.
\end{abstract}

Keywords: Salmonella, XDR S. Typhi, Azithromycin

\section{INTRODUCTION}

Salmonella $s p$. is a gram-negative bacillus that causes outbreaks of food-borne infections worldwide and carries high rates of morbidity and mortality, especially in developing countries [1]. Salmonella $s p$. infections often present with enteric fever, septicemia, a focal disease with or without bacteremia, gastroenteritis, and sometimes as chronic carrier state [2].

Typhoid fever still causes an estimated 200,000 deaths in low and middle-income countries each year [3].

The first-line antibiotics to treat typhoid include ampicillin, trimethoprim-sulfamethoxazole, and chloramphenicol [4]. S. Typhi strains resistant to these three antibiotics are considered multidrugresistant (MDR). These were first identified in the late 1970s to early 1980s [5]. MDR Salmonella infections are treated with either ceftriaxone, a third-generation cephalosporin, or quinolones. MDR Salmonellae that are resistant to these two groups of antibiotics are called extensively drug-resistant (XDR) [4]. Currently, azithromycin is the only remaining reliable and affordable first-line oral antibiotic for XDR typhoid in low-resource settings [6].

However, sporadic cases of ceftriaxone- or azithromycin-resistant $S$. Typhi have recently been reported $[7,8]$. We present a case of XDR $S$. Typhi that was also resistant to azithromycin in a suspected case of Endocarditis who responded to carbapenem therapy. The purpose of this case report is to highlight the importance of $S$. Typhi in patients with prolonged fever such as Endocarditis in our country. Moreover, the emergence of Azithromycin resistance in our country will lead to a therapeutic challenge to treat XDR S. Typhi infection.

\section{CASE}

A non-smoker non-addict non-diabetic, normotensive man in his twenties, operated for Aortic Valve Replacement and on warfarin therapy for the past 3 years presented on 19 April 2019 with deranged INR in the Emergency department of Rawalpindi Institute of Cardiology, a tertiary care cardiac hospital in twin capital city

Correspondence: Dr. Arif Maqsood Ali, Department of Pathology and Blood Bank, Rawalpindi Institute of Cardiology, Rawalpindi, Pakistan 
of Rawalpindi Islamabad, Pakistan. He had a history of moderate fever for 1 week. The fever was continuous that was managed with antipyretics. There was a history of mild anorexia and constipation. There was no history of nausea, vomiting, or abdominal pain. No history of any chest, abdominal, urinary, or other systemic complaints.

On examination, she was pale in appearance and had time, place, and person orientation. His pulse was 88 beats/minute, BP was $110 / 70 \mathrm{~mm}$ $\mathrm{Hg}$ and temperature was $99{ }^{0} \mathrm{~F}$. Electrocardiography and Echocardiography showed mild lateral wall ischemia. His tongue was coated. There was no visceromegaly. Systemic examination did not reveal any abnormality except for machinery like a murmur in the aortic area. Investigations showed prolonged Prothrombin time and INR which were $25.7 \mathrm{sec}$ and 2.38 respectively. Hemoglobin was $11.1 \mathrm{~g} / \mathrm{dl}$. The white cell count was $2.14 \times 109 / 1$ with $48 \%$ polymorphs, $37 \%$ lymphocytes, and $13 \%$ monocytes. The platelet count was 290x103/ul. ESR was $90 \mathrm{~mm}$ fall at the first hour. Mean Corpuscular Hemoglobin $(\mathrm{MCH})$ and Mean Corpuscular Volume (MCV) were 28 pg \& $82 \mathrm{fL}$ respectively. The red cell morphology was normocytic normochromic. Serum urea was $30 \mathrm{mg} / \mathrm{dl}$, creatinine $0.8 \mathrm{mg} / \mathrm{dl}$ and uric acid 9.4 $\mathrm{mg} / \mathrm{dl}$. C- reactive protein (CRP) was $68.2 \mathrm{mg} / \mathrm{L}$. Lipid profile showed total cholesterol of 240 $\mathrm{mg} / \mathrm{dl}$ and triglycerides $274 \mathrm{mg} / \mathrm{dl}$. Liver function tests showed Bilirubin $0.6 \mathrm{mg} / \mathrm{dl}, \mathrm{ALT} 178 \mathrm{U} / \mathrm{L}$ \& Alkaline phosphatase $145 \mathrm{U} / \mathrm{L}$. Serum electrolytes and cardiac enzymes were within normal limits. He was seronegative for $\mathrm{HBsAg}$, Anti $\mathrm{HCV}$, and HIV. Urine routine examination was normal and its culture did not yield any growth.

Three consecutive blood cultures were taken during fever and incubated in Bact Alert 3D (Biomeriux France). The system showed a positive signal after 3 days of incubation at 37 ${ }^{\circ} \mathrm{C}$ in all three blood culture bottles. These were subcultured on Blood and MacConkey agar which yielded growth of catalase-positive, oxidase negative, and non-lactose fermenting colonies (Figure 1). The growths were identified by Vitek 2 Compact (Biomerieux, France) as Salmonella serotype Typhi (Figure 2).

It was resistant to ampicillin, trimethoprimsulphamethoxazole, and chloramphenicol but susceptible to ertapenem, imipenem, and meropenem. The isolates were resistant to ciprofloxacin \& intermediately susceptible to levofloxacin. The susceptibility to azithromycin $(15 \mu \mathrm{g})$ was performed by Kirby- Bauer disc diffusion method (Figure 3). Results for azithromycin were interpreted as per the CLSI [7]. All three isolates of $S$. Typhi had inhibition zone sizes of $\leq 12 \mathrm{~mm}$ and were reported as resistant to azithromycin. The patient was administered meropenem parenterally. He became afebrile after 3 days and the antibiotic was continued for another 4 days. The patient was advised to continue to follow up in the cardiac outpatient department.

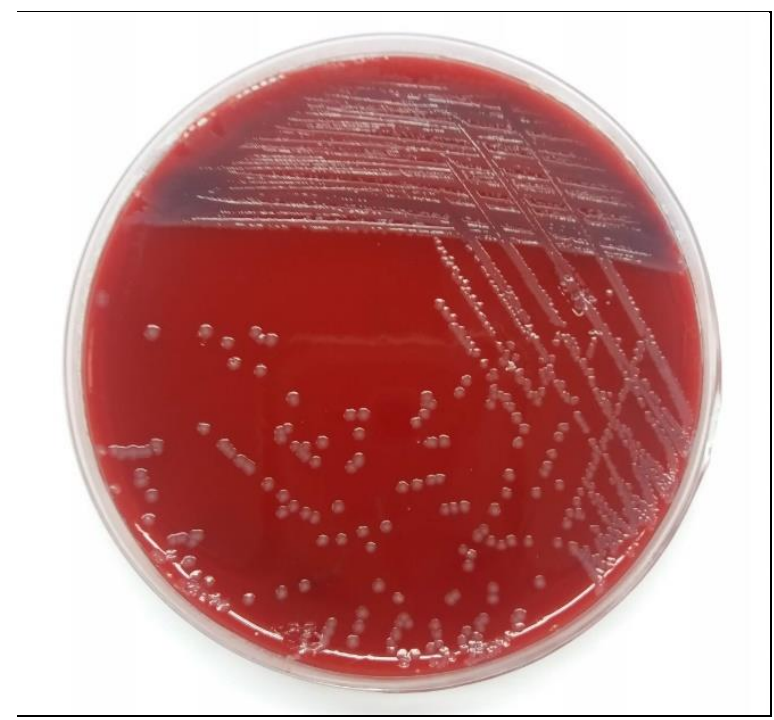

Figure 1. Growth of Salmonella Typhi on Blood Agar.

\section{DISCUSSION}

Typhoid fever is a serious food-borne infection caused by Salmonella enterica. The disease is endemic in our country due to contaminated water supplies and a lack of sanitary facilities.

More than 100,000 cases are reported annually [8]. MDR S. Typhi has been reported in several studies from all over Pakistan. $S$. Typhis resistance to chloramphenicol, ampicillin, and trimethoprim-sulfamethoxazole is labeled as MDR [9]. A recent study carried out in the capital city of Pakistan, Islamabad in 2017 showed 64 MDR S.

Typhi and five S. Paratyphi isolates out of 197 patients with typhoid fever. However, there was no resistance to cephalosporins [10]. A retrospective multicenter study was carried out at Armed Forces Institute of Pathology (AFIP), 
Rawalpindi from 2005 to 2014 showed frequent MDR \& Fluoroquinolone resistance in S. Typhi.

However, no XDR S. Typhi was isolated [11].

\begin{tabular}{|c|c|c|c|c|c|}
\hline $\begin{array}{l}\text { bioMérieux Customer: Micro Lab } \\
\text { Patient Name. } \\
\text { Location: } \\
\text { Lab ID: } 4882.1\end{array}$ & \multicolumn{5}{|c|}{$\begin{array}{r}\text { Printed Jan 8, } 2019 \text { 00:49 PKT } \\
\text { Patient ID } \\
\text { Physician: } \\
\text { Isolate Number: } 2 \\
\end{array}$} \\
\hline \multicolumn{6}{|c|}{$\begin{array}{l}\text { Organism Quantity: } \\
\text { Selected Organism : Salmonella ser.Typhi }\end{array}$} \\
\hline \multicolumn{5}{|l|}{ Source: } & Collected: \\
\hline \multicolumn{6}{|l|}{ Comments: } \\
\hline Susceptibility Information & \multicolumn{3}{|c|}{ Analysis Time: 12.15 hours } & Status: & Final \\
\hline \begin{tabular}{|c|} 
Antimicrobial \\
\end{tabular} & \begin{tabular}{|c|} 
MIC \\
\end{tabular} & Interpretation & Antimicrobial & MIC & Interpretation \\
\hline ESBL & & & Ertapenem & $<=0.5$ & $\mathrm{~s}$ \\
\hline Ampicillin & $>=32$ & $\mathrm{R}$ & Imipenem & $<=0.25$ & $\mathrm{~s}$ \\
\hline Amoxicillin/Clavulanic Acid & 16 & 1 & Meropenem & $<=0.25$ & $\mathrm{~s}$ \\
\hline Piperacillin/Tazobactam & 8 & $\mathrm{~s}$ & Gentamicin & $<=1$ & ${ }^{*} \mathrm{R}$ \\
\hline Cefazolin & $>=64$ & $\mathrm{R}$ & Ciprofloxacin & $>=4$ & $\mathrm{R}$ \\
\hline Ceftriaxone & $>=64$ & $\mathrm{R}$ & Levofloxacin & 4 & 1 \\
\hline Cefepime & $>=64$ & $\mathrm{R}$ & Tetracycline & 2 & $\mathrm{~s}$ \\
\hline Aztreonam & $>=64$ & $\mathrm{R}$ & Trimethoprim/Sulfamethoxazole & $>=320$ & $\mathrm{R}$ \\
\hline \multicolumn{6}{|c|}{$+=$ Deduced drug $\quad *=$ AES modified ${ }^{* *}=$ User modified } \\
\hline \multicolumn{6}{|l|}{ AES Findings } \\
\hline Confidence: & onsistent & & & & \\
\hline
\end{tabular}

Figure 2. Susceptibility of Third isolate by Kirby Bauer method on Mueller Hinton Agar.

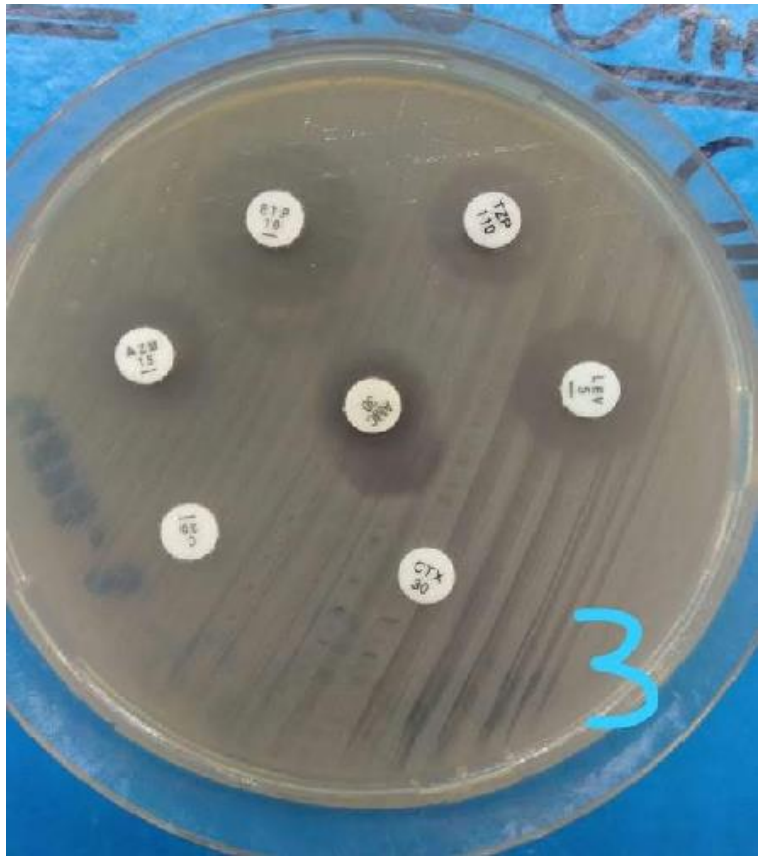

Figure 3. Susceptibility of Third isolate by Kirby Bauer method on Mueller Hinton Agar

A new XDR strain resistant to third-generation cephalosporins was first reported in 2016 in Hyderabad, Pakistan. It spread to 14 districts of Sindh province, infecting more than 8,000 people. XDR S. Typhi does not respond to thirdgeneration cephalosporins due to mutation of H58 haplotype [8]. From November 2016 to September 2017, 339 cases of XDR S. Typhi strain were reported in Pakistan, mostly in Karachi and Hyderabad; one travel-associated case was also reported from the United Kingdom. XDR Typhi strains show susceptibility to azithromycin and carbapenems [12].

We isolated an XDR $S$. Typhi in three consecutive cultures of a patient suspected of Endocarditis with prosthetic valve replacement. S. Typhi isolates were XDR and showed 
resistance to Azithromycin. The patient responded to injection meropenem therapy without complications. This is the first such case report of XDR S. Typhi resistant to azithromycin isolated in our country.

Typhoid patients are often treated with macrolide azithromycin in our country due to a lack of resistance to this antibiotic [6]. Increased use of azithromycin places selective pressure on the emergence of azithromycin-resistant isolates. There are a few sporadic reports on azithromycin treatment failures [13].

In Bangladesh between 2009 and 2016, twelve S. Typhi and one ParaTyphi A strains were found resistant to azithromycin in a surveillance study among indoor departments of the two largest pediatric hospitals [14].

Although azithromycin treatment failure in Salmonella Para Typhi A has been documented in a few case reports, however, no clinical and microbiological failure in $S$. Typhi has yet been reported [15].

The isolation of the first Azithromycin XDR $S$. Typhi in our case report is a whistleblower to the medical community to ensure rational use of antibiotics to treat Salmonella infections in our country to avoid its spread globally. Efforts should be made to improve hygiene and sanitation besides mass vaccination against typhoid.

\section{ACKNOWLEDGMENTS}

Financial Support: This study was performed without any financial support.

Conflict of interest: The authors have declared no conflicts of interest.

\section{REFERENCES}

1. Feasey NA, Dougan G, Kingsley RA, Heyderman RS, Gordon MA. Invasive non-typhoidal Salmonella disease: An emerging and neglected tropical disease in Africa. Lancet 2012; 379(9835):2489-99.

2. Sudhaharan S, Padmaja K, Solanki R, Lakshmi V, Umabala P, Aparna B. Extra-intestinal Salmonellosis in a tertiary care hospital in South India. J Infect Dev Ctries 2014; 8(7):831-7.

3. Crump JA, Luby SP, Mintz ED. The global burden of typhoid fever. Bulletin of the World Health Organization 2004; 82:346-53.

4. Crump JA, Sjölund-Karlsson M, Gordon MA, Parry CM. Epidemiology, clinical presentation, laboratory diagnosis, antimicrobial resistance, and antimicrobial management of invasive Salmonella infections. Clin Microbiol Rev 2015; 28(4):901937.

5. Olarte J, Galindo E. Salmonella Typhi resistant to chloramphenicol, ampicillin, and other antimicrobial agents: strains isolated during an extensive typhoid fever epidemic in Mexico. Antimicrob Agent Chemother 1973; 4(6):597-601.

6. Klemm EJ, Shakoor S, Page AJ, et al. Emergence of an extensively drug-resistant Salmonella enterica serovar Typhi clone harboring a promiscuous plasmid encoding resistance to fluoroquinolones and third-generation cephalosporins. MBio 2018; 9(1): e00105-18.

7. Clinical and Laboratory Standards Institute. Performance standards for antimicrobial susceptibility testing. CLSI, Wayne 2018; 28th ed: document M100.

8. Bhatti JM, Memon Y, Sarfaraz S, Salahuddin N. An unusual case of extensively drug resistant typhoid fever. Cureus 2019;11(5).

9. Qamar FN, Yousafzai MT, Khalid M, et al. Outbreak investigation of ceftriaxone-resistant Salmonella enterica serotype Typhi and its risk factors among the general population in Hyderabad, Pakistan: a matched case-control study. The Lancet Infectious Diseases 2018; 18(12):1368-76.

10. Morgenstern R, Hayes PC: The liver in typhoid fever: always affected, not just a complication. Am J Gastroenterol 1991; 86:1235-9.

11. Ramanathan M: Unusual hepatic manifestations in typhoid fever. Singapore Med J 1991; 32:335-7.

12. Chatham-Stephens K, Medalla F, Hughes M, et al. Emergence of Extensively Drug-Resistant Salmonella Typhi Infections Among Travelers to or from Pakistan-United States, 2016-2018. Morbidity and Mortality Weekly Report 2019; 68(1):11.

13. Molloy A, Nair S, Cooke FJ, et al. First report of Salmonella enterica serotype paratyphi A azithromycin resistance leading to treatment failure. Journal of clinical microbiology 2010; 48(12):4655-4657.

14. Hooda Y, Saha S, Sajib MS, et al. Emergence and molecular basis of azithromycin resistance in typhoidal Salmonella in Dhaka, Bangladesh. bioRxiv 2019; 1:594531.

15. Manesh A, Balaji V, Kumar DR, Rupali P. A case of clinical and microbiological failure of azithromycin therapy in Salmonella enterica serotype Typhi despite low azithromycin MIC. Intern J Infect Dis 2017; 54:62-63. 